\title{
Spectroscopic Study of Cepheids in the Globular Cluster Omega Cen
}

\author{
Guillermo Gonzalez and George Wallerstein \\ Department of Astronomy, FM-20, \\ University of Washington, Seattle WA 98195
}

\begin{abstract}
Until now there have been few spectroscopic studies of cepheids in globular clusters. In this preliminary report we present sample spectra of the stars V1 and V29 in $\omega$ Cen. Eventually, we hope to use the abundance patterns, masses, and period changes of cepheids to better understand post-horizontal branch evolution in globular clusters.
\end{abstract}

\section{Introduction}

On the asymptotic giant branch (AGB) a star begins to experience thermal pulses due to the unstable nature of a double shell energy source, leading to "blue loops" on the HR diagram. Blue loops can explain the occurence of the W Vir and RV Tau variables in globular clusters. Lower luminosity BL Her variables are believed to be evolving from the hot side of the HB to the AGB, while at least some of the RV Tau variables are probably post-AGB stars. Globular clusters such as $\omega$ Cen with several cepheids and only one likely post-AGB star may provide the "rosetta stones" needed to understand post-HB stellar evolution. Since many globular clusters have well determined parameters including distance, age, metallicity, and reddening, the analysis of the composition and evolutionary status of the cepheids and related stars in globulars is more secure than the analysis of field stars.

\section{Goals}

We have undertaken this investigation with the following goals in mind: (1) to find out at what point in a star's post-HB life s-process and CNO elements reach the surface; (2) to calculate the amount of mixing between the envelope and the interior; (3) to calculate the mean neutron exposure of the s- process elements; (4) to look for possible fractionation effects; (5) to measure the masses of the cepheids from CCD multicolor photometry and spectroscopic data; and (6) to calculate the helium abundance from the $\mathrm{He}$ I emission lines. 

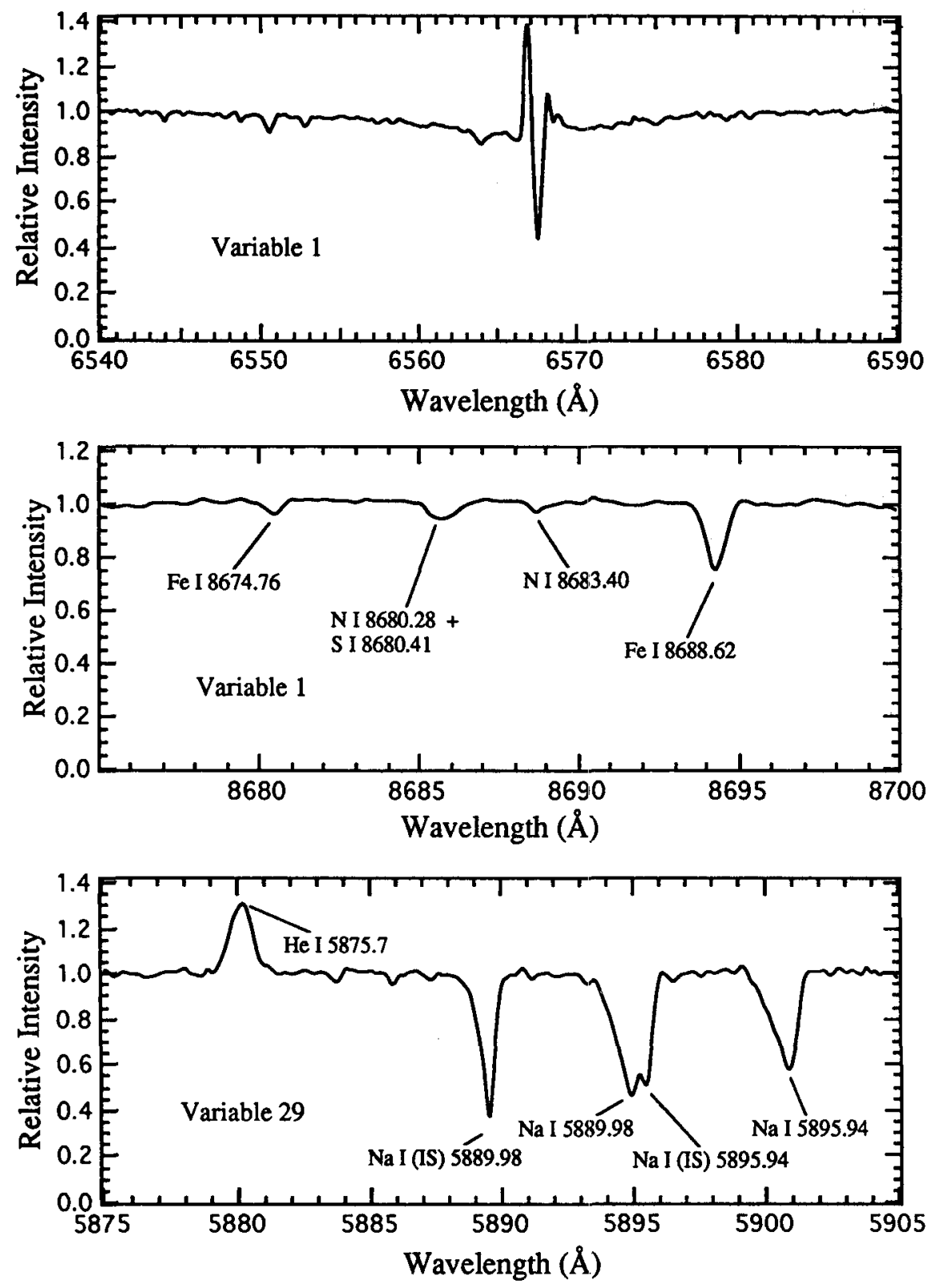

Figure 1. (Top) Spectrum of V1 obtained during rising light. The blue-shifted emission is produced by the shock in the atmosphere. The red-shifted absorption is produced by cooler infalling layers above the shock. (Middle) Infrared region of the spectrum of V1 showing some of the N I and S I lines. (Bottom) Spectrum of V29 showing one of the He I emission lines (visible during rising light) and the photospheric and interstellar $\mathrm{Na} I$ lines. 